\title{
Construction Of Wavelet Bases That Mimic The Behaviour Of Some Given Operator
}

\author{
Ildar Khalidov, Dimitri Van De Ville, Thierry Blu, Michael Unser \\ Biomedical Imaging Group, Ecole Polytechnique Fédérale de Lausanne (EPFL), Switzerland
}

\begin{abstract}
Probably the most important property of wavelets for signal processing is their multiscale derivative-like behavior when applied to functions. In order to extend the class of problems that can profit of wavelet-based techniques, we propose to build new families of wavelets that behave like an arbitrary scale-covariant operator. Our extension is general and includes many known wavelet bases. At the same time, the method takes advantage a fast filterbank decomposition-reconstruction algorithm. We give necessary conditions for the scale-covariant operator to admit our wavelet construction, and we provide examples of new wavelets that can be obtained with our method.
\end{abstract}

Keywords: Wavelets, splines, differential operators, Green's functions, continuous-time signal processing, multiresolution approximation, multiresolution analysis

\section{INTRODUCTION}

Modeling of the surrounding world often is done in terms of continuous domain. Operators, such as the Laplacian or the gradient, could be used to extract interesting features (e.g., edges) from continuous-domain models. At the same time, continuous calculus is computationally prohibitive, and therefore can not be directly used in practice.

A common approach in signal processing is to use sampled data and a discrete approximation of the operator. The obvious advantage is the speed of computations - applying a shift-invariant operator to the data corresponds to simple filtering. However, this approach offers almost no control over approximation error.

To combine the advantages of the continuous and the discrete point of view, signals can be represented with (discrete) coefficients in (continuous) multi-resolution pyramid of linear shift-invariant (LSI) spaces. In this approach, the signal, most often represented by its samples, is projected on the LSI space at some initial scale, and all the following treatment is done on its coefficients. The important question is then the choice of convenient spaces that are adapted for the problem at hand.

In signal analysis, we are typically interested in particular features, and often those can be detected by continuous operators. These operators could be considered as signal "decorrelators". For example, edges are well detected by the derivative in 1-D or by the laplacian in 2-D. A 1-D edge, represented with a step function, turns into a Dirac delta-function, whose position indicates the location of the edge.

In the past decades, wavelets gained particular interest, as they provide a complete and stable multiscale representation of $L_{2}$, and coefficients in the wavelet basis correspond to the samples of the (iterated) derivative of the smoothed signal. At the same time, the wavelet decomposition is very efficient from the computational point of view due to the fast filtering algorithm.

In this paper, we construct wavelets that behave like a given operator L. In our approach, the multiresolution spaces are completely characterized by the Green function of the operator. Importantly, the operator-like wavelet can be constructed directly from the operator, bypassing the scaling function space. What makes the approach even more attractive, is that the wavelet space is generated by the shifts of a single wavelet function. Our work provides a nice generalization of some known and used constructions like elliptic wavelets, ${ }^{1}$ Laplacianlike polyharmonic spline wavelets ${ }^{2}$ and exponential-spline wavelets. ${ }^{3}$ We give necessary (and nearly sufficient) conditions on the operator L to be wavelet-admissible.

Further author information: (ildar.khalidov, dimitri.vandeville, thierry.blu, michael.unser)@epfl.ch 
A model signal that gets fully "decorrelated" by L, could be constructed from Dirac delta functions using the inverse operator $\mathrm{L}^{-1}$. The behavior of such a signal is, to a large extent, defined by the configuration of the discontinuities of $\mathrm{L}^{-1}$. In this paper, we restrict ourselves to the case of pole-type discontinuities. The key result of the present work is that our wavelet's behavior at the analysis matches closely that of the underlying operator near the poles. In our decomposition, the wavelet coefficients at the scale $i$ can be considered as samples of $\mathrm{L}\left\{\phi_{i} * f\right\}$, where $\phi_{i}$ is a smoothing kernel.

\section{PRELIMINARIES}

Consider a linear, shift-invariant operator L that acts on the class of functions $f: \mathbb{R}^{d} \rightarrow \mathbb{C}$, where $d$ is the dimension parameter. The operator $\mathrm{L}$ is characterized by its Laplace transform $L(\mathbf{s})$ or, equivalently, by its frequency response $\hat{L}(\boldsymbol{\omega})=L(j \omega)$.

DEFINITION 2.1. L is of order $r$ if and only if, for all positive $\rho<r-d / 2$, we have that

$$
\sum_{\mathbf{n} \in \mathbb{Z}^{d}} \frac{\|\boldsymbol{\omega}+2 \pi \mathbf{n}\|^{2 \rho}}{1+|\hat{L}(\boldsymbol{\omega}+2 \pi \mathbf{n})|^{2}} \leq C_{\rho}<\infty
$$

Essentially, Definition 2.1 requires the frequency response $\hat{L}(\boldsymbol{\omega})$ of the operator to grow at least at the rate of $\|\boldsymbol{\omega}\|^{r}$ as $\|\boldsymbol{\omega}\| \rightarrow \infty$. The associated Sobolev space is defined by

$$
W_{2}^{\mathrm{L}}=\left\{f \in L_{2}\left(\mathbb{R}^{d}\right): \int_{\mathbb{R}^{d}}|\hat{f}(\boldsymbol{\omega})|^{2}\left(1+|\hat{L}(\boldsymbol{\omega})|^{2}\right) d \boldsymbol{\omega}<\infty\right\} .
$$

DEFINITION 2.2. L is spline-admissible of order $r$ if and only if the following conditions are satisfied: ${ }^{4}$

1. L is a linear shift-invariant operator of smoothness order $r>d / 2$;

2. $\mathrm{L}$ has a well-defined inverse $\mathrm{L}^{-1}$, and its impulse response $\rho(\mathbf{x})$ of $\mathrm{L}^{-1}$ is a function of slow growth. Thus, $\rho$ is a Green function of $\mathrm{L}: \mathrm{L}\{\rho(\mathbf{x})\}=\delta(\mathbf{x})$;

3. There exists a localization operator $\hat{\Delta}\left(e^{j \boldsymbol{\omega}}\right)=\sum_{\mathbf{k} \in \mathbb{Z}^{d}} p[\mathbf{k}] e^{-j\langle\boldsymbol{\omega}, \mathbf{k}\rangle}$ with $p \in \ell_{1}\left(\mathbb{Z}^{d}\right)$ such that the corresponding generalized B-spline $\varphi(\mathbf{x})=\Delta\{\rho\}(\mathbf{x})$ satisfies the Riesz basis condition.

Suppose now that we have a family of linear, shift-invariant operators $\left\{\mathrm{L}_{\vec{\nu}}\right\}$, indexed by the parameter vector $\vec{\nu}=\left(\nu_{1}, \ldots, \nu_{L}\right)$.

DEFINITION 2.3. The operators $\mathrm{L}_{\vec{\nu}}$ are called scale-covariant if, for each a $>0$, their transfer functions satisfy

$$
L_{a \vec{\nu}}(a \mathbf{s})=c(a) \cdot L_{\vec{\nu}}(\mathbf{s}),
$$

where $c(a)$ depends only on the scale $a$. In other words, the family $\left\{\mathrm{L}_{\vec{\nu}}\right\}$ is invariant with respect to arbitrary scaling.

In the examples, we will use the unit step function $u(x)=(1+\operatorname{sign}(x)) / 2$ and the rectangle fucntion $\operatorname{rect}(x)=u\left(x+\frac{1}{2}\right)-u\left(x-\frac{1}{2}\right)$.

EXAMPLE 1. Let $d=1$ and $\mathrm{L}_{\nu}=\mathrm{D}-\nu \mathrm{I}$, where $\nu<0$ is a parameter, $\mathrm{D}$ is the derivative and $\mathrm{I}$ is the identity operator. Let us verify that $\mathrm{L}_{\nu}$ is spline-admissible and scale-covariant. Indeed, $\mathrm{L}_{\nu}$ is of order $r=1>\frac{1}{2}$, and its inverse has an impulse response $\rho_{\nu}(x)=e^{\nu x} u(x)$. With the localization operator $\hat{\Delta}_{\nu}\left(e^{j \omega}\right)=1-e^{j \omega-\nu}$, we obtain the first-order exponential B-spline

$$
\varphi_{\nu}(x)=\rho_{\nu}(x)-e^{\nu} \rho_{\nu}(x-1)
$$

which is a function of compact support. We show this function in Fig.1. 


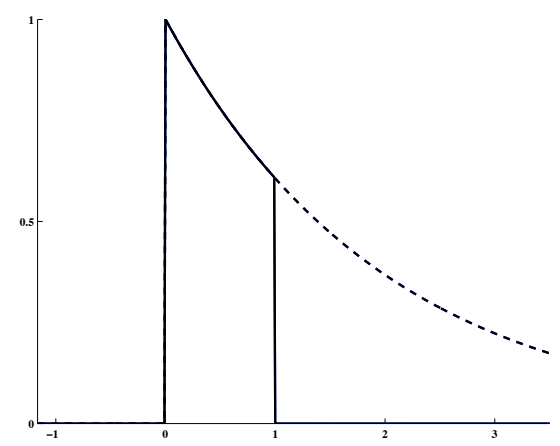

Figure 1. The generalized B-spline (solid line) is obtained by localization of the operator's Green function (dashed line).

Furthermore, $\hat{L}_{\nu}(\omega)$ satisfies $\hat{L}_{a \nu}(a \omega)=a j \omega-a \nu=a \hat{L}_{\nu}(\omega)$, which proves that $\mathrm{L}_{\nu}$ is scale-covariant with $c(a)=a$.

The class of scale-covariant operators is large. All scale-invariant operators are scale-covariant, and therefore included. At the same time, the definition (2.3) allows us to apply our results to more general operator families, such as Matérn operators $\hat{L}_{\nu}(\boldsymbol{\omega})=\|\boldsymbol{\omega}\|^{2}+\nu^{2}$ and differential operators with rational transfer functions.

For the remaining part of this paper, we suppose that $\left\{\mathrm{L}_{\vec{\nu}}\right\}$ is a family of scale-covariant, spline-admissible operators of order $r$. We assume that the poles of the operator are bounded; i.e., there exists $M>0$ such that for any $\mathbf{p} \in \mathbb{R}^{d}$ with $\|\mathbf{p}\|>M$, we have $\hat{L}(\mathbf{p}) \neq 0$. The scale $a$ will take the values $a=m^{i}$, where $m>0$ is the integer dilation factor; for notational simplicity, we will omit the index $\vec{\nu}$ and use $i$ to label the corresponding operators and functions.

\section{MULTIRESOLUTION ANALYSIS}

Consider the function $s_{i}(\mathbf{x})$ of smoothness order $r$, which is characterized by the relation

$$
\mathrm{L}\left\{s_{i}\right\}(\mathbf{x})=\sum_{\mathbf{k} \in \mathbb{Z}^{d}} c_{\mathbf{k}} \delta\left(\mathbf{x}-m^{i} \mathbf{k}\right)
$$

We call this function an L-spline with knots $m^{i} \mathbf{k}$. Clearly, $s_{i}(\mathbf{x})$ satisfies

$$
s_{i}(\mathbf{x})=\sum_{\mathbf{k} \in \mathbb{Z}^{d}} c_{\mathbf{k}} \rho\left(\mathbf{x}-m^{i} \mathbf{k}\right)+p(\mathbf{x})
$$

where $\operatorname{L}\{p\}(\mathbf{x})=0$; i.e., the function $p(\mathbf{x})$ belongs to the null-space of the operator L.

Remember that the operator $\mathrm{L}$ admits a generalized B-spline $\varphi_{i}(\mathbf{x})=\Delta_{i}\{\rho\}(\mathbf{x})$. We define the multiresolution space $V_{i}$ by plugging $\varphi_{i}$ at the locations $m^{i} \mathbf{k}, \mathbf{k} \in \mathbb{Z}^{d}$ and taking all linear combinations that are in $L_{2}$ :

$$
V_{i}=\left\{s(\mathbf{x}): s(\mathbf{x})=\sum_{\mathbf{k} \in \mathbb{Z}^{d}} c[\mathbf{k}] \varphi_{i}\left(\mathbf{x}-m^{i} \mathbf{k}\right), c[\mathbf{k}] \in l_{2}\left(\mathbb{Z}^{d}\right)\right\}
$$

In this paper, we assume that $V_{i}$ consists of all L-splines that are in $L_{2}$; this requires that some Strang-Fix-like conditions on the localization operator $\Delta_{i}$ are fulfilled. ${ }^{5}$ By definition, we have $V_{i} \subset V_{i-1}$. The condition on the operator order $r>d / 2$ ensures that the error rate $\left\|P_{i} f-f\right\|_{2} \rightarrow 0$ as $i \rightarrow-\infty$ for any $f \in W_{2}^{r}$, where $P_{i}$ is the orthogonal projector on $V_{i}$. Consequently, the multiresolution analysis $\cup V_{i}$ is dense in $L_{2}$.

By extending a similar result for one-dimensional operators, ${ }^{4}$ it is straightforward to show that $\mathrm{L}^{*} \mathrm{~L}$ is a spline-admissible operator of order $2 r>d>d / 2$. Its corresponding B-spline, which is given by $\varphi_{i}(\mathbf{x}) * \varphi_{i}^{*}(-\mathbf{x})$, generates a Riesz basis. Consequently, the $\mathrm{L}^{*} \mathrm{~L}$-spline interpolant $\left.\phi_{i}(\mathbf{x})\right)$, given by

$$
\left.\phi_{i}(\mathbf{x})\right) \leftrightarrow \hat{\phi}_{i}(\boldsymbol{\omega})=\frac{\left|\hat{\varphi}_{i}(\boldsymbol{\omega})\right|^{2}}{\sum_{\mathbf{k} \in \mathbb{Z}^{d}}\left|\hat{\varphi}_{i}\left(\boldsymbol{\omega}+2 \cdot m^{-i} \pi \mathbf{k}\right)\right|^{2}},
$$


is well-defined and generates a Riesz basis. Importantly, $\phi_{i} \in W_{2}^{\mathrm{L}}$ does not depend on the specific choice of the localization operator $\Delta_{i}$, as we can see from

$$
\hat{\phi}_{i}(\boldsymbol{\omega})=\frac{\left|\hat{\Delta}_{i}\left(e^{j m^{i} \boldsymbol{\omega}}\right)\right|^{2} /|\hat{L}(\boldsymbol{\omega})|^{2}}{\left|\hat{\Delta}_{i}\left(e^{j m^{i} \boldsymbol{\omega}}\right)\right|^{2} \sum_{\mathbf{k} \in \mathbb{Z}^{d}} 1 /\left|\hat{L}\left(\boldsymbol{\omega}+2 \cdot m^{-i} \pi \mathbf{k}\right)\right|^{2}}=\frac{1}{1+|\hat{L}(\boldsymbol{\omega})|^{2} \sum_{\mathbf{k} \in \mathbb{Z}^{d} \backslash \mathbf{0}} 1 /\left|\hat{L}\left(\boldsymbol{\omega}+2 \cdot m^{-i} \pi \mathbf{k}\right)\right|^{2}} .
$$

The $\mathrm{L}^{*} \mathrm{~L}$-spline interpolant plays the key role in our wavelet construction, which we describe in the next section.

\section{OPERATOR-LIKE WAVELETS}

Let us fix the scale $i$. We construct the generating wavelet function as

$$
\psi_{i+1}=\mathrm{L}^{*}\left\{\phi_{i}\right\}
$$

where we can apply the operator $\mathrm{L}^{*}$ because $\phi_{i} \in W_{2}^{\mathrm{L}}$. The Fourier domain expression for $\hat{\psi}_{i+1}$ has the form

$$
\hat{\psi}_{i+1}(\boldsymbol{\omega})=\frac{\hat{L}^{*}(\boldsymbol{\omega})}{1+|\hat{L}(\boldsymbol{\omega})|^{2} \sum_{\mathbf{k} \in \mathbb{Z}^{d} \backslash \mathbf{0}} \frac{1}{\left|\hat{L}\left(\boldsymbol{\omega}+2 \cdot m^{-i} \pi \mathbf{k}\right)\right|^{2}}} .
$$

Note that for every $\mathbf{p} \in \mathbb{R}^{d}$ such that $\hat{L}^{*}(\mathbf{p})=0, \hat{\psi}_{i+1}(\boldsymbol{\omega})$ vanishes at $\mathbf{p}_{1}+2 \pi \mathbf{k} / m^{i}$. In other words, each pole of $\mathrm{L}$ generates a periodic sequence of zeros in the generating wavelet's spectrum.

The wavelet system is obtained by shifting $\psi_{i+1}$ to all coset points $m^{i} \mathbb{Z}^{d} \backslash m^{i+1} \mathbb{Z}^{d}$ of the dilated grid. Remarkably, we have only one unique wavelet function even when the dimension $d$ is bigger than one; the $m^{d}-1$ wavelets $\psi_{i+1}^{(1)}, \ldots, \psi_{i+1}^{\left(m^{d}-1\right)}$ are shifts of $\psi_{i+1}$.

Let us study the properties of the new wavelet system.

Property 1. The wavelets $\left\{\psi_{i+1}\left(\mathbf{x}-m^{i} \mathbf{k}\right)\right\}_{k \in m^{i} \mathbb{Z}^{d} \backslash m^{i+1} \mathbb{Z}^{d}}$ are orthogonal to the space $V_{i+1}$.

Proof. It is sufficient to prove that $\left\langle\rho(\mathbf{x}), \psi_{i+1}\left(\mathbf{x}-m^{i} \mathbf{k}\right)\right\rangle=0$ for all $k \in m^{i} \mathbb{Z}^{d} \backslash m^{i+1} \mathbb{Z}^{d}$. Passing the conjugate operator to the left side of the scalar product, we have

$$
\left\langle\rho(\mathbf{x}), \psi_{i+1}\left(\mathbf{x}-m^{i} \mathbf{k}\right)\right\rangle=\left\langle\mathrm{L} \rho\left(\mathbf{x}+m^{i} \mathbf{k}\right), \phi_{i}(\mathbf{x})\right\rangle=\left\langle\delta\left(\mathbf{x}+m^{i} \mathbf{k}\right), \phi_{i}(\mathbf{x})\right\rangle=0,
$$

as $\phi_{i}$ is the interpolant.

We conclude that $\left\{\psi_{i+1}\left(x-m^{i} \mathbf{k}\right)\right\}_{k \in m^{i} \mathbb{Z}^{d} \backslash m^{i+1} \mathbb{Z}^{d}, i \in \mathbb{Z}}$ is a semi-orthogonal wavelet system.

PROPERTy 2. The wavelet function $\psi_{i+1}$ behaves like a multiscale version of the underlying operator $\mathrm{L}$ in the sense that, for any $f \in W_{2}^{r}$, we have $f * \psi_{i+1}^{T}=\mathrm{L}\left\{f * \phi_{i}^{T}\right\}$.

The proof of this property is straightforward with our wavelet construction. For a vast majority of operators, $\phi_{i}$ is a lowpass filter with its passband varying accordingly to the scale. Therefore, $\left\{\mathrm{L}\left\{f * \phi_{i}^{T}\right\}\right\}_{i \in \mathbb{Z}}$ corresponds to the multiscale version of $\mathrm{L}$.

The next property gives the necessary condition on the operator that the corresponding operator-like wavelet system is Riesz-stable.

Property 3. Let $i \in \mathbb{Z}$ be an arbitrary scale. If $\left\{\psi_{i+1, \mathbf{k}}\right\}_{\mathbf{k} \in \mathbb{Z}^{d}}$ is a Riesz basis, then, for each $\mathbf{p} \in \mathbb{R}^{d}$ such that $\hat{L}(\mathbf{p})=0$,

$$
\hat{L}\left(\mathbf{p}+2 \pi \mathbf{l} / m^{i}+2 \pi \mathbf{k}_{0} / m^{i+1}\right) \neq 0, \mathbf{k} \in \mathbb{Z}^{d}, \mathbf{k}_{0} \in\{0,1\}^{d} \backslash \mathbf{0} .
$$

Proof. We use the result of de Boor et $\mathrm{al}^{6}$ and construct the system's Gram matrix $G_{i}(\boldsymbol{\omega})=\left[g_{i}^{m n}(\boldsymbol{\omega})\right]$ with

$$
g_{i}^{m n}=\sum_{\mathbf{k} \in \mathbb{Z}^{d}}\left(\hat{\psi}_{i+1}^{(m)} \cdot \hat{\psi}_{i+1}^{(n) *}\right)\left(\boldsymbol{\omega}+2 \pi \mathbf{k} / m^{i+1}\right) .
$$


The determinant of this matrix should be separated from zero and bounded for all $\boldsymbol{\omega}$. This is possible if and only if the $m^{d}-1$ "fibers" $\left(\hat{\psi}_{i+1}^{(m)}\left(\boldsymbol{\omega}+2 \pi \mathbf{k} / m^{i+1}\right)_{\mathbf{k} \in \mathbb{Z}^{d}}\right)$ are linearly independent. In our case, the fibers have particularly simple structure: they are $m$-periodic $d$-dimensional sequences, multiplied by $\hat{\psi}_{i+1}(\boldsymbol{\omega})$.

Suppose that $\mathrm{L}$ violates the necessary condition; i.e., there exist $\mathbf{p}_{1}, \mathbf{p}_{2} \in \mathbb{R}^{d}$ such that $\hat{L}\left(\mathbf{p}_{1}\right)=\hat{L}\left(\mathbf{p}_{2}\right)=0$ and $\mathbf{p}_{1}=\mathbf{p}_{2}+2 \pi \mathbf{l} / m^{i}+2 \pi \mathbf{k}_{0} / m^{i+1}$. Then, $\hat{\psi}_{i+1}\left(\mathbf{p}_{1}\right)=\hat{\psi}_{i+1}\left(\mathbf{p}_{1}+2 \pi \mathbf{k}_{0} / m^{i+1}\right)=0$. For $\boldsymbol{\omega}=\mathbf{p}$, the $m^{d}-1$ fibers will have two zeros on their main period at the positions corresponding to $\mathbf{k}=\mathbf{0}$ and $\mathbf{k}=\mathbf{k}_{0}$. The size of the main period is $m^{d}$, so the fibers are linearly dependent.

The condition (2) is close to sufficient; however, the exact formulation of the latter is slightly technical and will be published elsewhere. Instead, let us study several examples that illustrate the richness of our wavelet construction.

EXAMPLE 2. For the first-order differential operator $\mathrm{L}_{\nu}=\mathrm{D}-\nu \mathrm{I}$ from Example 1, the autocorrelation filter is identity. Therefore, we have $\sum_{\mathbf{k} \in \mathbb{Z}^{d}}\left|\hat{\varphi}_{i}\left(\omega+2 \cdot m^{-i} \pi \mathbf{k}\right)\right|^{2}=1$ and the $\mathrm{L}^{*} \mathrm{~L}$-interpolant $\phi_{i}(x)$ is a symmetric exponential spline with poles $\left\{\nu,-\nu^{*}\right\}$. As $d=1$ and $m=2$, the wavelet space is generated by a single shift of the wavelet function, which is the exponential-spline wavelet (see Figure 2).

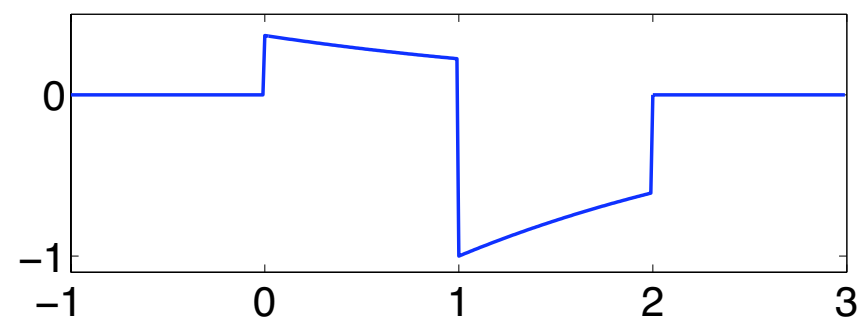

Figure 2. First order exponential-spline wavelet corresponds to the choice of $\mathrm{L}_{\nu}=\mathrm{D}-\nu \mathrm{I}$.

EXAmple 3. With $m=3$ and the derivative operator $\mathrm{L}=\mathrm{D}$, we get the wavelet system, generated by two Haar wavelets, shifted to the points $x=1$ and $x=2$. Interestingly, despite the apparent simplicity of this operator, the separable extension to multiple dimensions is not possible - for example, the 2-D operator $\mathrm{D}_{x} \mathrm{D}_{y}$ fails the necessary stability condition.

ExAmple 4. The 2-D Matérn operator $\hat{L}_{\nu}(\boldsymbol{\omega})=\|\boldsymbol{\omega}\|^{2}+\nu^{2}$ does not have poles. Therefore, no localization operator is needed for its Green's function. We show the corresponding wavelet in Figure 3. The function has sharp peaks, which are caused by slow decay of the wavelet spectrum in the Fourier domain.

EXAmple 5. As $\nu \rightarrow 0$, the Matérn operator tends to the scale-invariant Laplacian operator $\hat{\Delta}(\boldsymbol{\omega})=\|\boldsymbol{\omega}\|^{2}$. The corresponding wavelet is shown in Fig. 4. In case of iterated Laplacian operator $\hat{\Delta}^{2}(\boldsymbol{\omega})=\|\boldsymbol{\omega}\|^{4}$, faster Fourier decay of the wavelet spectrum leads to better regularity in space domain (see Fig. 5).

\section{CONCLUSION}

We have constructed wavelet-like bases that behave like the multiresolution version of a given scale-covariant, spline-admissible operator. In our construction, all wavelets are shifts of a single generating function. In the multidimensional setting, the wavelets are not separable. In general, separable wavelets can not be obtained with this non-separable construction.

From the computational point of view, our wavelets still admit fast Mallat's filterbank algorithm, although, the filters are generally scale-dependent. Whenever the transform is used intensively, it might be a reasonable option to precompute these filters and store them in memory to accelerate computations.

Our wavelet bases have potential of application in areas of signal processing, where one is dealing with data convolved with a known imaging operator $\mathrm{L}^{-1}$. The wavelets derived from this operator would behave like a multiscale version of $\mathrm{L}$ and essentially decorrelate the data, concentrating the object's energy into a small number of coefficients. 


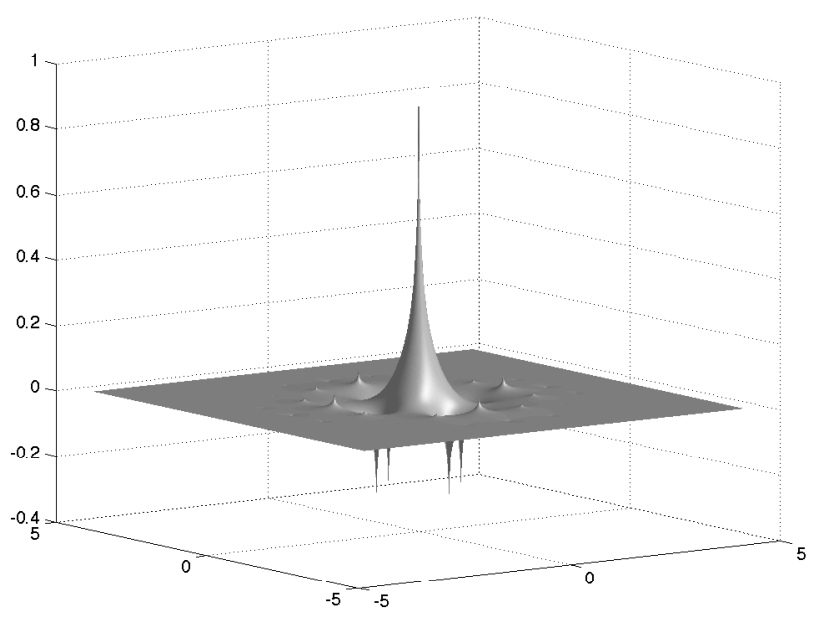

Figure 3. Wavelet obtained from the Matérn operator; $\nu=1$.

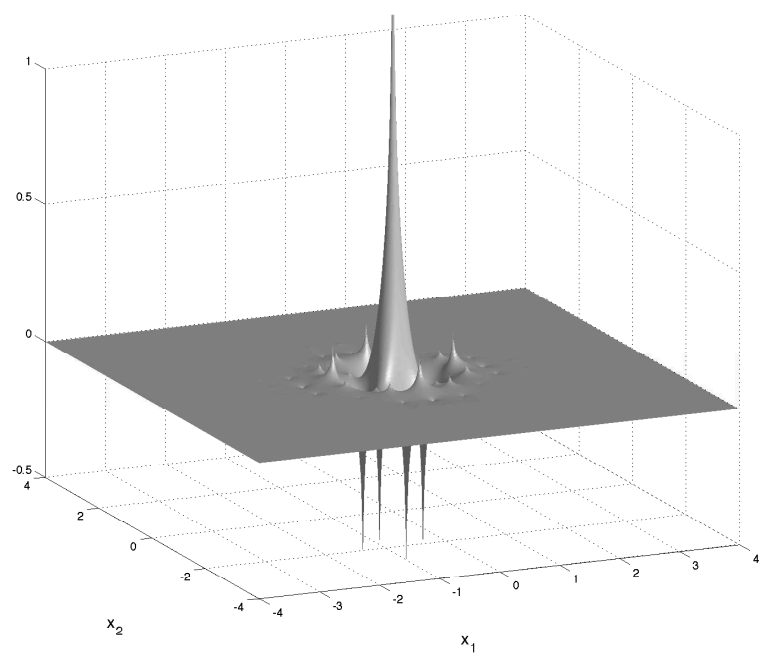

Figure 4. Wavelet obtained from the Laplacian operator. 


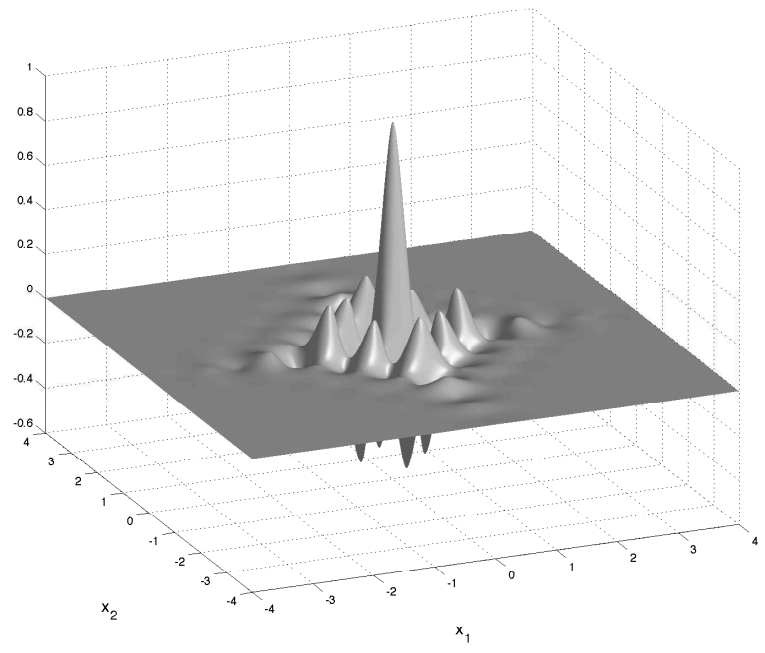

Figure 5. Wavelet obtained from the iterated Laplacian operator.

\section{ACKNOWLEDGMENTS}

This work was supported by the Center for Biomedical Imaging (CIBM) of the Geneva - Lausanne Universities and the EPFL, the foundations Leenaards and Louis-Jeantet, as well as by the Swiss National Science Foundation under grant 200020-109415.

\section{REFERENCES}

1. C. Micchelli, C. Rabut, and F. Utreras, "Using the refinement equation for the construction of pre-wavelets III: Elliptic splines," Numerical Algorithms 1, October 1991.

2. D. Van De Ville, T. Blu, and M. Unser, "Isotropic polyharmonic b-splines: Scaling functions and wavelets," IEEE Trans. Image Process. 14, pp. 1798-1813, November 2005.

3. I. Khalidov and M. Unser, "From differential equations to the construction of new wavelet-like bases," IEEE Trans. Signal Process. 54, pp. 1256-1267, April 2006.

4. M. Unser and T. Blu, "Generalized smoothing splines and the optimal discretization of the Wiener filter," IEEE Trans. Signal Process. 53, pp. 2146-2159, June 2005.

5. I. Khalidov, T. Blu, and M. Unser, "Generalized L-spline wavelet bases," in Proceedings of the SPIE Conference on Mathematical Imaging: Wavelet XI, 5914, pp. 59140F-1-59140F-8, (San Diego CA, USA), July 31-August 3, 2005.

6. C. De Boor, R. DeVore, and A. Ron, The structure of finitely generated shift-invariant spaces in $L_{2}\left(\mathbb{R}^{d}\right)$, University of Wisconsin-Madison, 1992. 